\title{
Photometric distances to young stars in the inner Galactic disk
}

\section{The region towards the open cluster Trumpler 27 at $L=355^{\circ \star, \star \star, \star \star \star}$}

\author{
G. Perren ${ }^{1}$, R. A. Vázquez ${ }^{2}$, and G. Carraro ${ }^{3,4}$ \\ ${ }^{1}$ Instituto de Física de Rosario, IFIR (CONICET-UNR), Parque Urquiza, 2000 Rosario, Argentina \\ e-mail: perren@ifir-conicet.gov.ar \\ 2 Facultad de Ciencias Astronómicas y Geofísicas (UNLP), Instituto de Astrofísica de La Plata (CONICET, UNLP), \\ Paseo del Bosque s/n, La Plata, Argentina \\ e-mail: rvazquez@fcaglp.unlp.edu.ar \\ 3 ESO, Alonso de Cordova 3107, Casilla 19100 Santiago de Chile, Chile \\ e-mail: gcarraro@eso.org \\ ${ }^{4}$ Dipartimento di Astronomia, Universita' di Padova, Vicolo Osservatorio 5, 35122 Padova, Italy
}

Received 23 May 2012 / Accepted 17 September 2012

\begin{abstract}
Context. The spiral structure of the Milky Way inside the solar circle is still poorly known because of the high density of the material that causes strong extinction towards the Galactic center.

Aims. We present results of the first extensive and deep color-color diagram (CCD) photometric survey carried out in the field of the open cluster Trumpler 27, an object immersed in a region of extremely high visual absorption in the constellation of Sagittarius not far from the Galaxy center. The survey covers almost a quarter of square degree.

Methods. We look for young stars clumps that might plausibly be associated with spiral structure. Wide-field UBVI photometry combined with infrared information allows us to reconstruct the distribution in the reddening and distance of young stars in the field using the CCD and color-magnitude diagrams (CMD).

Results. The analysis of our data, combined with extensive spectroscopy taken from the literature, shows that the real entity of Trumpler 27 as an open cluster is far from being firmly stated. In fact, instead of finding a relatively compact group of stars confined to a small distance range, we found that stars associated with Trumpler 27 are, indeed, a superposition of early-type stars seen along the line of sight extending over several kiloparsecs beyond even the center of the Galaxy. We demonstrate that at each distance range it becomes possible to generate a CMD resembling that of an open cluster. This way, our analysis indicates that what was considered an open cluster characterized by a significant age spread is a stellar continuum that reaches its maximum number of stars at approximately $3.5 \mathrm{kpc}$ from the Sun, the distance of the Scutum-Crux arm approximately. After analyzing the way early-type stars distribute with distance, we found that some of these stellar groups may be linked, within the distance errors, with other inner spiral arms of our Galaxy, including the Near $3 \mathrm{kpc}$ arm at approximately $5 \mathrm{kpc}$ from the Sun. However, very young stars by themselves do not seem to trace strongly the inner spiral arms since they are distributed evenly across several kiloparsecs toward the center of the Galaxy. This is remarkably different to current $\mathrm{HI}$ and $\mathrm{CO}$ radio observation maps, which show inner spiral arms composed by discrete structures of gas with a well-defined inter-arm separation.
\end{abstract}

Key words. Galaxy: disk - open clusters and associations: general - open clusters and associations: individual: Trumpler 27 stars: early-type - Galaxy: structure

\section{Introduction}

One of the authors (Carraro 2011) has very recently examined the parameters of young diffuse stellar populations in the direction $l=314^{\circ}$ as seen in the background of some open clusters and related them successfully with the inner Galactic structure. This study made use of photometric techniques that we developed in the last decade and successfully applied to the third quadrant of the Milky Way (Carraro et al. 2005; Moitinho et al. 2006; Vázquez et al. 2008).

* Based on observations collected at Las Campanas Observatory, Chile.

$\star \star$ Full photometric data and Tables 2 and 3 are only available at the CDS via anomymous ftp to cdsarc.u-strasbg.fr (130.79.128.5) or via

http://cdsarc.u-strasbg.fr/viz-bin/qcat?]/A+A/548/A125

$\star \star \star$ Table 1 is available in electronic form at http://www . aanda.org
In Carraro (2011, hereafter Paper I) of the series, we extensively described the project and the employed methods and proceeded to study the spatial distribution of early-type stars (spectral types $\mathrm{O}$ and $\mathrm{B}$ ) in the direction of $l=314^{\circ}$. Apart from the prominent Carina-Sagittarius spiral arm, we detected for the first time signatures of the Scutum-Crux arm in that direction. These findings confirmed the predictions of earlier studies on the location and pitch angles of part of the spiral arm pattern in the fourth Galactic quadrant.

The present article, the second of the series, is aimed at improving our knowledge of the spiral structure in the inner Galactic disk in another direction, much closer to the Galaxy center, at $l=355^{\circ}$. In this case, instead of a single pointing as in Paper I, we combined three pointings to cover a wide region ( $\sim 25$ arcmin on a side) in the direction of Trumpler 27. This is an open cluster located in the Sagittarius constellation, five degrees from the center of the Galaxy and slightly below the Galactic 
plane, at coordinates $\alpha_{2000}=17^{\mathrm{h}} 36^{\mathrm{m}} 20^{\mathrm{s}}, \delta_{2000}=-33^{\circ} 31^{\prime}$ ( $\left.l=355^{\circ} .064, b=-0.742\right)$. The zone occupied by the cluster is heavily obscured by dust clouds, but nevertheless it is still possible to see a significant but sparse group of relatively bright stars. The first extensive photometric survey on this object was conducted by The \& Stokes (1970), who obtained UBV photographic photometry for about 40 stars spread over a circular area of $11^{\prime}$ radius around the assumed center of this cluster. They found huge values for the color excess $E_{(B-V)}$, ranging from approximately -0.6 to $2.5 \mathrm{mag}$. Despite this, they found that the extinction law is normal with $R_{V}=\frac{A_{V}}{E_{B-V}}=3.1$ and placed the cluster at a heliocentric distance of $1.1 \mathrm{kpc}$. Later on, Moffat et al. (1977, hereinafter MFJ77) conducted a deeper $U B V$ photometric survey, including photoelectric observations of the brightest stars and photographic ones for the rest. This study was combined with spectroscopic analysis for a significant number of stars (about 50) and suggested that Trumpler 27 is at a distance of $2.1 \pm 0.2 \mathrm{kpc}$ from the Sun with almost similar reddening values to the ones previously computed by The \& Stokes (1970). Bakker \& The (1983) used Walraven photometric data combined with infrared and $U V B$ photometry. This last study confirmed that the extinction law is normal (i.e. $R_{V}=3.1-3.2$ ), and that the cluster is located at a distance of $1.7 \pm 0.25 \mathrm{kpc}$ and is about $10^{7}$ years old.

After a 20-year hiatus with no investigations, Trumpler 27 was the target of a new spectroscopic campaign carried out by Massey et al. (2001, hereinafter MDEW01) where the oldest spectral types were verified and spectroscopy was made for several stars that had not previously been measured. This new spectroscopic survey revealed a surprisingly hot and massive stellar population in the area of this object: one M supergiant, ten supergiants of B-types, two Wolf-Rayet stars, one Of-type star, two O-type giant stars, other stars of B-types and one G0I, probably a Cepheid star. In MDEW01, the parameters of several stars in Trumpler 27 were redetermined on the basis of this new spectral survey but retaining the old $U V B$ photometry made by MFJ77. In this investigation, the authors found that the cluster is located at a distance larger than any previous determination: $2.5 \mathrm{kpc}$ from the Sun. The next interesting finding made by MDEW01 is the detection of a significant age spread among the B-type stars. It appears that some of them show ages ranging from 6 to $10 \times 10^{6}$ years and are about 2 to $4 \times 10^{6}$ older than the rest of them. They assume that this age spread comes from the large difficulty in separating cluster members from background stars in this highly obscured zone.

Given its position close to the Galaxy center and on the Galactic plane, Trumpler 27 is an ideal target for the purpose of investigating part of the spiral structure in the fourth Galactic quadrant. Its surroundings contain a large number of potential blue stars, some of which are considered excellent tracers of spiral arms, such as W-R stars and O-type stars as shown by MDEW01 and MFJ77. It is useful to recall that it has been almost 30 years since the last photometric study on this object and that we are now in the position to take advantage of the large number of spectral types to re discuss the distance and age of Trumpler 27 with modern $U B V I$ observations. These two parameters are of paramount importance because this cluster may become a good Galactic spiral arm tracer if the distance discrepancy of the order of about $1.4 \mathrm{kpc}$ from one author to the other is removed. In other words, such a difference means that the cluster may belong to the Carina-Sagittarius arm or the ScutumCentaurus arm. But first we should assess whether Trumpler 27 really exists and if the distance discrepancies found are only due to the treatment of the strong reddening of the region and/or to the fact that in clusters as heavily obscured as this one, only the vertical upper main sequence is useful for fitting a reference line (e.g., the ZAMS of Schmidt-Kaler 1982).

In Sect. 2, we discuss the data acquisition, the reduction process, the stellar astrometry, and the comparison with former photometry in the region. In Sect. 3, we show and discuss the main photometric diagrams and introduce the strategy to derive intrinsic parameters of stars in Trumpler 27. In Sect. 4, we analyze the dataset derived for hot stars and the nature of Trumpler 27. Section 5 includes a description of findings using infrared data. In Sect. 6, we collect the evidence to interpret the structure of the inner Galaxy as seen through the Trumpler 27 region. Conclusions are given in Sect. 7.

\section{Observation and data reduction}

Three regions containing Trumpler 27 were observed in the $U B V I$ photometric system at Las Campanas Observatory (LCO) on four different runs in 2006, 2010, and 2011, as illustrated in Table 1, which lists useful details of the observations, like filter coverage, airmass range, and exposure time and sequences. We used the SITe\#3 color-color diagram (CCD) detector onboard the Swope $1.0 \mathrm{~m}$ telescope ${ }^{1}$. With a pixel scale of 0.435 arcsec/pixel, this CCD allowed us to cover $14.8 \times 22.8$ arcmin on sky. In all run, the seeing was good, ranging from 0.9 arcsec to 1.8. However, post-processing indicated that in the 2006 and 2010 runs the nights were not completely photometric, and for this reason we only rely on the 2011 standard stars. The whole field of view is shown in Fig. 1, a montage of all $V$ exposure images, and covers an area of $26 \times 23$ squared arcmin. North is up, and east to the left. To determine the transformation from our instrumental system to the standard Johnson-Kron-Cousins system and to correct for extinction, we observed stars in Landolt's areas PG 1323, PG 1633, and MarkA (Landolt 1992) multiple times and with different airmasses ranging from $\sim 1.07$ to $\sim 2.0$ and covering quite a large color range $-0.3 \leq(B-V) \leq 1.7$ mag. All the images from previous runs were then shifted to the 2011 run by means of all common stars before transforming the photometry into the standard system.

\subsection{Basic photometric reduction}

Basic calibration of the CCD frames was done using $\mathrm{IRAF}^{2}$ package CCDRED. For this purpose, zero exposure frames and twilight sky flats were taken every night. All frames were pre reduced, applying trimming, bias, and flat-field correction. Before flat-fielding, all frames were corrected for linearity, following the recipe discussed in Hamuy et al. (2006).

Photometry was then performed using the IRAF DAOPHOT/ALLSTAR and PHOTCAL packages. Instrumental magnitudes were extracted following the point-spread function (PSF) method (Stetson 1987). A quadratic, spatially variable, master PSF (PENNY function) was adopted because of the large field of view of the detector. Aperture corrections were then determined, making aperture photometry of a suitable number (typically 15 to 20) of bright, isolated stars in the field.

\footnotetext{
1 http://www.1co.cl/telescopes-information/ henrietta-swope/

2 IRAF is distributed by the National Optical Astronomy Observatory, which is operated by the Association of Universities for Research in Astronomy, Inc., under cooperative agreement with the National Science Foundation.
} 
G. Perren et al.: Photometric distances to young stars in the inner Galactic disk. II.

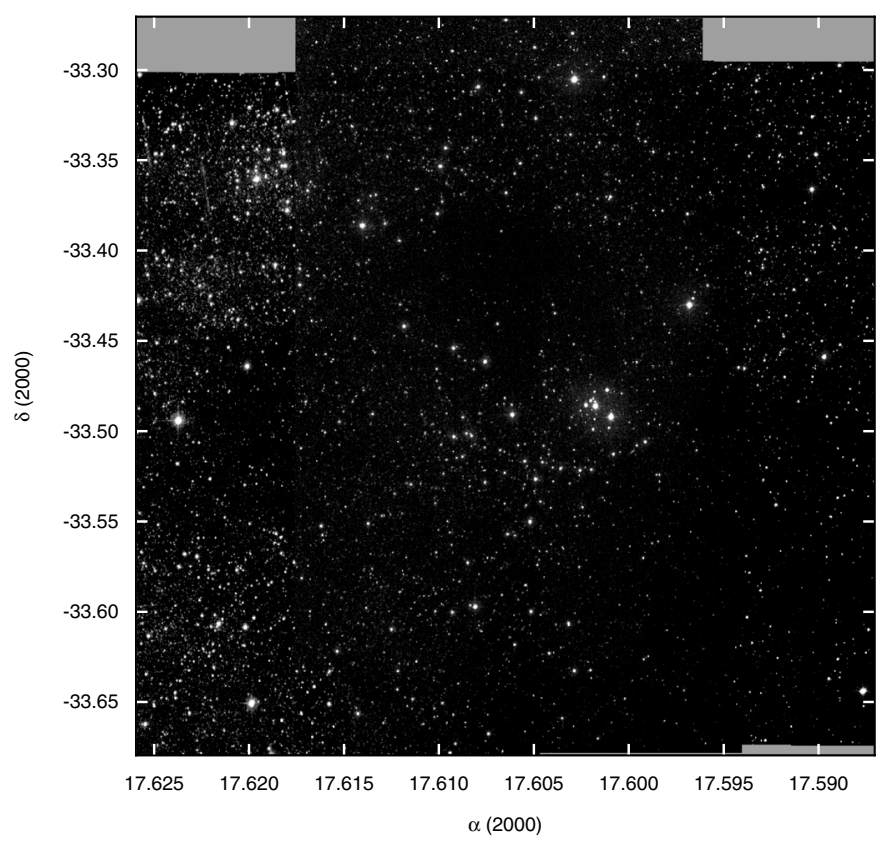

Fig. 1. Montage of all $V$ images (28) taken in the area of Trumpler 27. The field is about $26 \operatorname{arcmin} \times 23$ arcmin. The $\alpha$ and $\delta$ coordinates are given in decimal notation. North is up, east to the left.

These corrections were found to vary from 0.160 to 0.290 mag, depending on the filter. The PSF photometry was finally aperture corrected, filter by filter.

\subsection{Photometric calibration}

After removing problematic stars and stars having only a few observations in Landolt's (1992) catalog, our photometric solution for the run was extracted by combining measures from both nights (after checking that they were stable and similar), yielding a grand total of 63 measurements per filter. It turned out to be

$$
\begin{aligned}
U= & u+(5.004 \pm 0.010)+(0.49 \pm 0.010) \times X \\
& +(0.129 \pm 0.013) \times(U-B) \\
B= & b+(3.283 \pm 0.006)+(0.25 \pm 0.010) \times X \\
& +(0.040 \pm 0.008) \times(B-V) \\
V= & v+(3.204 \pm 0.005)+(0.16 \pm 0.010) \times X \\
& -(0.066 \pm 0.008) \times(B-V) \\
I= & i+(3.508 \pm 0.005)+(0.08 \pm 0.010) \times X \\
& +(0.037 \pm 0.006) \times(V-I),
\end{aligned}
$$

where $X$ indicates the airmass. The final rms of the fitting in this case was $0.040,0.019,0.015$, and 0.015 in $U, B, V$, and $I$, respectively.

Global photometric errors were estimated using the scheme developed by Patat \& Carraro (2001, Appendix A1), which takes into account the errors resulting from the PSF fitting procedure (i.e., from ALLSTAR) and the calibration errors (corresponding to the zero point, color terms, and extinction errors). In Fig. 2, we present our global photometric errors in $V,(B-V),(U-B)$, and $(V-I)$ plotted as a function of $V$ magnitude. Quick inspection shows that stars brighter than $V \approx 20$ mag have errors lower than $\sim 0.05$ mag in magnitude and lower than $\sim 0.10 \mathrm{mag}$ in $(B-V)$ and $(V-I)$. Higher errors, as expected, are seen in $(U-B)$. The final catalog contains 9769 UBVI entries.

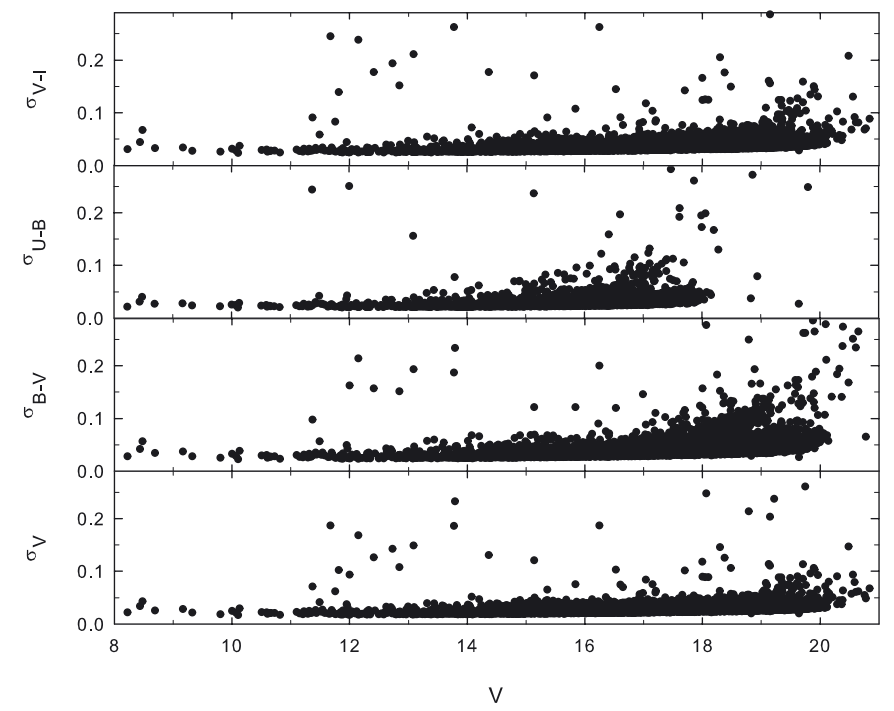

Fig. 2. Trend of global photometric errors in magnitude and colors as a function of our $V$ magnitude. See text for details.

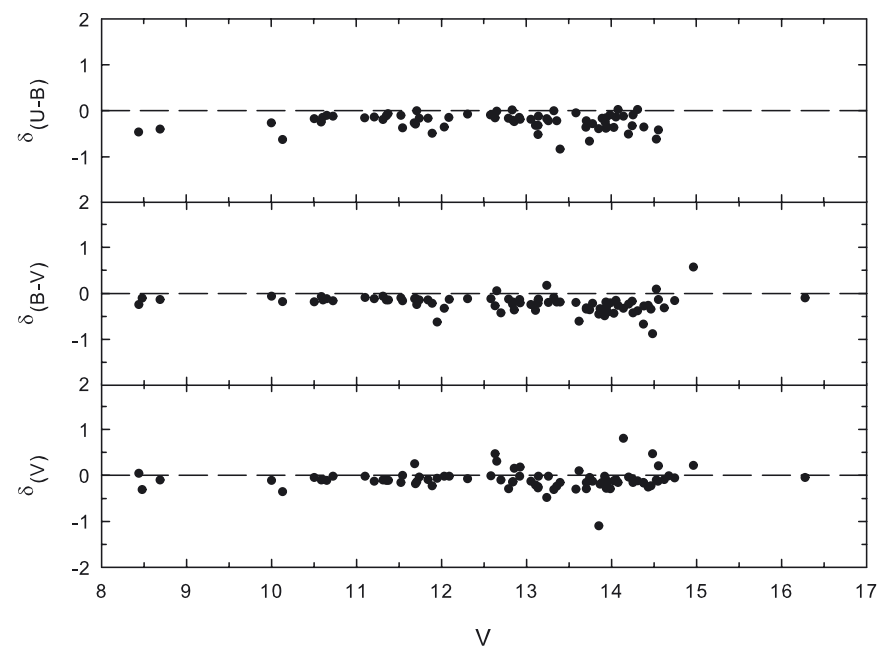

Fig. 3. Differences of our photometry with MFJ77 plotted against our $V$ magnitudes. Upper panel, $\delta_{U-B}$, mid panel $\delta_{B-V}$, lower panel $\delta_{V}$.

\subsection{Astrometry}

The optical catalogue was cross-correlated with 2MASS, which resulted in a final catalog including $U B V I$ and $J H K_{\mathrm{s}}$ magnitudes. As a by-product, pixel (i.e., detector) coordinates were converted to RA and Dec for J2000.0 equinox, thus providing 2MASSbased astrometry, useful for, e.g., spectroscopic follow-up. The rms of the residuals in the positions were 0.15 , which is about the astrometric precision of the 2 MASS catalog $\left(0{ }^{\prime} .12\right.$, Skrutskie et al. 2006).

\subsection{Comparison with former photometry}

Up to now, the most extensive catalog of $U B V$ photometry in the region of Trumpler 27 belongs to MFJ77. The other photometric sets are only photographic or in the Walraven system. Comparing our data set with MFJ77, we found 83 common stars in $V, 81$ in $(B-V)$, and 68 in $(U-B)$. The differences in the sense of our data minus MFJ77, are shown in Fig. 3 as a function of our $V$. Most of the stars in the MFJ77 survey have photographic photometry, and just a few have photoelectric photometry. In the 
comparison process, we found at least half a dozen stars showing strong differences in apparent magnitude and/or color indices. This may be produced, especially in crowded regions, in an unavoidable star-light contamination by nearby neighbors when using diaphragms in photoelectric measures. Mean differences and standard deviations are $\overline{\delta_{V}}=-0.08 \pm 0.15, \overline{\delta_{(B-V)}}=-0.19 \pm 0.15$ and $\overline{\delta_{(U-B)}}=-0.23 \pm 0.17$. The systematic trend in Fig. 3, in the sense that our values are brighter and bluer than those of MFJ77 and the large scatter, is likely produced by some zero point differences between our photometry and MFJ77's photographic and photoelectric photometry made with different telescopes and different diaphragm apertures, respectively.

\section{The all-area photometric diagrams}

Earlier CCDs and color-magnitude diagrams (CMDs) of the region of Trumpler 27 have been always very difficult to interpret because of strong and variable internal reddening and the consequent difficulties in defining the upper main sequence. Besides, the upper part of Trumpler 27 CMD is quite vertical and short, which makes any fitting of the ZAMS (zero age main sequence, Schmidt-Kaler 1982) dubious. The sum of these difficulties has precluded this object from a more in-depth study so far (McSwain \& Giess 2005).

Figure 4 shows the CCD (upper left-hand panel), the $V$ vs. $(B-V)$ (upper right-hand panel), the $V$ vs. $(U-B)$ (lower lefthand panel) and the $V$ vs. ( $V-I)$ (lower right-hand panel) CMDs. The solid line in the CCD is the ZAMS of Schmidt-Kaler (1982) shown in the normal $R_{V}=3.1$ position. The reddening path for a B5-type star is also indicated. A simple visual inspection of the CCD makes it possible to establish three dominant stellar populations:

- a first one, above the reddening line for a typical B5-type star, composed undoubtedly of blue stars strongly affected by variable reddening;

- a second stellar population appears below the reference line for a B5 star (the yellowish zone), composing a sort of stellar band starting at the second knee of the ZAMS and extending to the red side because of different amounts of reddening affecting it. This large star concentration corresponds, photometrically speaking, to A- and early F-type stars but also to reddened $\mathrm{B}$-type stars later than $\mathrm{B} 5$, as indicated by the path of the reddening in the CCD;

- finally, a third stellar population (greenish color) little affected by reddening appears extending from the domain of A to $\mathrm{M}$ stars all along the intrinsic reference line. Most of these stars are likely to be nearby objects since visual absorption maps from Neckel \& Klare (1980) reveal a sudden absorption increment at less than $1 \mathrm{kpc}$ in this direction. Therefore, the detection of intrinsically faint stars not reddened or just marginally affected by interstellar dust is only possible if they are close enough to the Sun.

Inspection of Fig. 1 lends further support to this scenario: intense absorption is seen at the west-southwest and immediately north of the center of Trumpler 27 and also to the east. A clear star overdensity is seen, in the same figure, at the northeast and at the expected location of the cluster core.

The CMDs ( $V$ vs. $(B-V), V$ vs. $(U-B)$ and $V$ vs. $(V-I))$ are less illustrative since not much can be extracted from them beyond the presence, above $V=16 \mathrm{mag}$, of an extended upper stellar sequence with a high degree of color dispersion that reflects
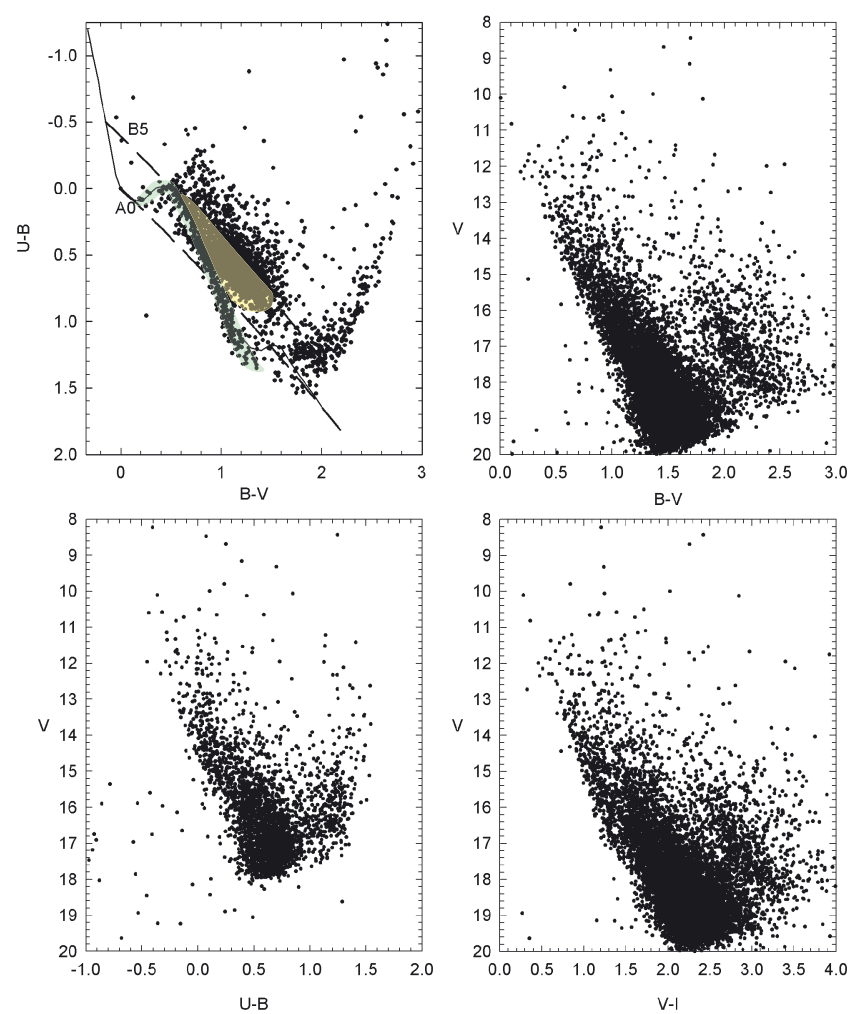

Fig. 4. The CCD, upper left panel, and the CMDs for $(B-V)$, upper right panel, for $(U-B)$, lower left panel, and for $(V-I)$, lower right panel, of all observed stars. Solid line in the CCD is the Schmidt-Kaler (1982) intrinsic line. Dashed lines in the CCD mean the path of the reddening for a B5- and an A0-type star as indicated by the labels. The bluish region represents the location of nearby dwarf stars; the yellowish one points out the locus occupied by A-F-type stars affected by increasing reddening. Stars above the B5 reddening line are young stars (see text), except those ones for which no reddening solution is possible.

the strong influence of dust in the zone. Since the three populations discussed previously are undistinguishable from each other in the CMDs, we estimate this type of diagram plays a marginal role in inferring useful information on this region.

\subsection{Deriving the parameters of early-type stars}

If the CMD is segmented with magnitude intervals and the corresponding $\mathrm{CCD}$ to each interval is built, it becomes easier to understand the way stellar populations develop in a given zone. The foundation and applications of this kind of analysis in open clusters and Galactic fields is developed in Carraro et al. (2007, 2009). In the present case, the procedure is shown in the series of CCD in Fig. 5 at intervals of 1 mag down to $V=18$. The superimposed lines follow the slope $E_{(U-B)}=$ $0.72 \times E_{(B-V)}+0.05 \times E_{(B-V)}^{2}$ explained long ago by Hiltner \& Johnson (1956) (corresponding to $R_{V}=3.1$ ) for stars of various spectral types from $\mathrm{O}$ to $\mathrm{B} 5$ in addition to the A-type stars.

The series of panels shows the color range covered by the three above-mentioned star populations. Actually, reddened hot stars along the reddening lines for $\mathrm{O}$ and B4 types look well detached from the rest down to $V \sim 14$. A comparison of our CCD with the ones produced by Bakker \& The (1983) in their Fig. 3 or in Fig. 5 of MFJ77 suggests that the major difference, apart from the inclusion of many faint stars, rests in the high number of new hot and reddened stars detected in our field which in turn is larger than the others. 
G. Perren et al.: Photometric distances to young stars in the inner Galactic disk. II.

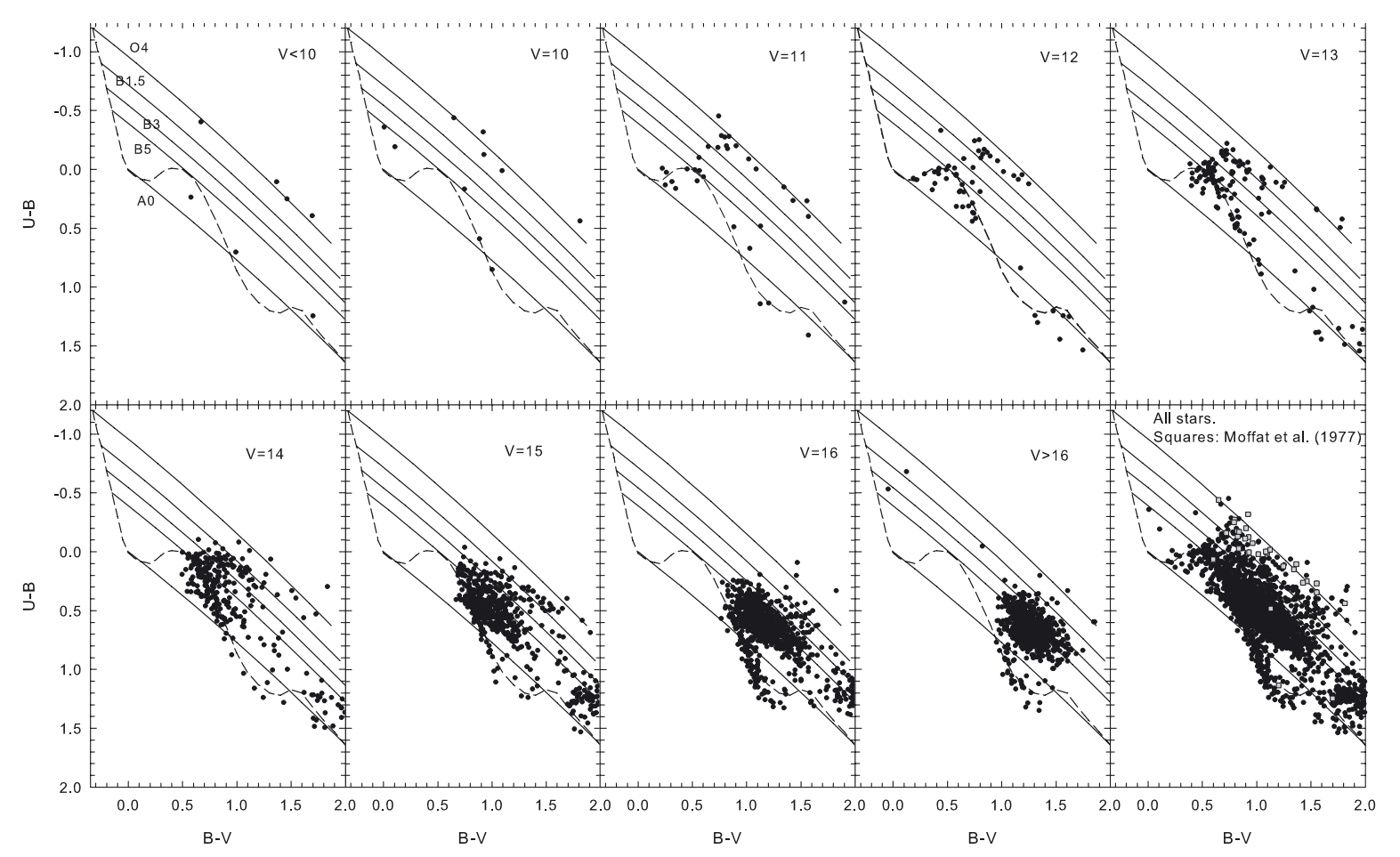

Fig. 5. The CCDs of the segmented CMD at intervals of 1 mag. Dashed line shows the position of the Schmidt-Kaler (1982) intrinsic line. The succession of solid lines show the path of the reddening for some of the early type stars indicated with the respective labels. The panel in the right lower corner shows the overall stars and those with spectral types, gray squares, from MFJ77.

The panel in the right-hand lower corner shows the overall $\mathrm{CCD}$, where we have identified, with open squares, bright stars already studied in previous articles via spectral analysis.

From $V \sim 14$, a stellar group (yellowish color) composed of a wide strip of A- and F-type stars becomes evident, reaching the maximum at $V=15$. Certainly, this latter group shows an ample range of reddening. Beyond this limit, dwarf stars of $\mathrm{G}-\mathrm{K}$ - and even some M-type stars are detected at an increasing number all along the intrinsic line, indicating that they are not so much affected by reddening and belong to the greenish zone. As stated above, these stars and part of the less reddened A-F-stars in the group belong to a nearby population in the line of sight to Trumpler 27.

In the rest of this paper, we shall focus on those stars above the reddening line for B5-type stars as indicated in Fig. 5 to derive individual distances by means of the spectroscopic parallax method since we know that they have unambiguous reddening solution in the CCD. This method has proved to be very efficient to approximate spectral types from photometry alone when unique reddening solutions are available as shown in Ahumada et al. (2011). Intrinsic colors for each star in this zone of the CCD were derived using the reddening line slope $E_{(U-B)}=0.72 \times E_{(B-V)}+0.05 \times E_{(B-V)}^{2}$ along with the relation $(U-B)_{0}=3.69 \times(B-V)_{0}+0.02$ (Carraro et al. 2010). This gives a second order equation where the $(B-V)_{0}$ index is the positive root and the $(U-B)_{0}$ index comes from the above expression. This locates the star on a point in the ZAMS (Schmidt-Kaler 1982) where the nearest spectral type is assigned. Unlike the Q-method that derives the $(B-V)_{0}$ star color assuming a linear relation of color excesses, the quadratic relation that we adopt here provides a more accurate solution when the color excess is high. Once individual color excesses and intrinsic colors are derived, we proceed to assign spectral types and absolute magnitudes following the relationship given by Schmidt-Kaler (1982). In this procedure, all stars are assumed to be of luminosity class V. However, a good number of hot stars have spectral types and luminosity class from MDEW01 and MFJ77. In these cases, the assignment of intrinsic color and absolute magnitude depends on the spectral type and luminosity class found by these authors using again the Schmidt-Kaler (1982) calibrations. Individual distance moduli are given after correcting apparent magnitudes by $A_{V}=3.1 \times E_{(B-V)}$. We accept $R=3.1$, which is completely in line with the rigorous study of the absorption throughout Walraven and infrared photometry developed by Bakker \& The (1983) in the region.

The distances obtained are photometric and subject therefore to uncertainties depending primarily on photometric errors. As done in Carraro (2011), we analyze the impact of these errors on distances by starting with the well-known expression for the distance modulus

$V-M_{V}=-5+5 \times \log (d)+A_{V}$.

A simple error propagation leads to

$\epsilon_{(d)}=\ln (10) \times d \times 0.2 \times\left[\sigma_{V}+3.1 \times \sigma_{(B-V)}\right]$,

assuming that $\epsilon_{\left(A_{V}\right)}=3.1 \times \epsilon_{(B-V)}$, where $\sigma_{(V)}$ and $\sigma_{(B-V)}$ directly come from photometry. In the final error computation we adopt $\epsilon_{\left(M_{V}\right)}=0$.

The results of this procedure yielded about 600 stars with intrinsic parameters and distances as indicated in Tables 2 and 3 in a self-explanatory format. Inspecting the errors in distance in the same tables, it follows that typical errors are about $20 \%$, quite reasonable for the method and of the same order of the ones obtained by the usual ZAMS (e.g., Schmidt-Kaler 1982) superposition. We want to emphasize that the assignment of spectral types 
Table 2. Intrinsic photometric values, distances and spectral types of stars in Trumpler 27.

\begin{tabular}{lcccccccrl}
\hline \hline Id & $E_{(B-V)}$ & $(B-V)_{0}$ & $(U-B)_{0}$ & $M_{V}$ & $d$ & $\sigma_{d}$ & ST $^{1}$ & Id(MFJ77) & ST $^{2}\left(\mathrm{MFJ77}_{,}\right.$MDEW01) \\
\hline 5517 & 1.69 & -0.28 & -1.00 & -2.60 & 3.17 & 0.73 & B0.5 & $(\ldots)$ & $(\ldots)$ \\
4732 & 1.10 & -0.28 & -1.00 & -2.60 & 2.64 & 0.58 & B0.5 & 19 & OB- \\
5355 & 1.14 & -0.21 & -1.02 & -6.90 & 6.60 & 0.29 & B0.5Ia & 16 & B0.5Ia \\
6456 & 1.12 & -0.02 & -0.62 & -7.10 & 6.74 & 0.15 & B0Ia & 2 & B0Ia \\
5672 & 1.80 & -0.27 & -0.95 & -2.35 & 3.38 & 0.78 & B1 & $(\ldots)$ & $(\ldots)$ \\
\hline
\end{tabular}

Notes. Id first column is our star numbering. Id column nine is the MFJ77 numbering. $\mathrm{ST}^{1}$ indicates the photometric spectral type found in this paper. $\mathrm{ST}^{2}(\mathrm{MFJ} 77, \mathrm{MDEW} 01)$ indicates spectral types from the literature.

Table 3. Idem for stars outside Trumpler 27.

\begin{tabular}{lcccrcclrl}
\hline \hline Id & $E_{(B-V)}$ & $(B-V)_{0}$ & $(U-B)_{0}$ & $M_{V}$ & $d$ & $\sigma_{d}$ & ST $^{1}$ & $\operatorname{Id}(\mathrm{MFJ77})$ & $\mathrm{ST}^{2}(\mathrm{MFJ77,} \mathrm{MDEW01)}$ \\
\hline 442 & 1.22 & -0.25 & -0.90 & -2.10 & 3.41 & 0.84 & $\mathrm{~B} 1.5$ & $(\ldots)$ & $(\ldots)$ \\
4497 & 1.09 & -0.25 & -0.90 & -2.80 & 2.83 & 1.33 & B1.5:V: & 32 & B1.5:V: \\
3629 & 1.15 & -0.26 & -0.95 & -3.20 & 3.22 & 0.95 & B1:V: & 34 & B1:V: \\
8932 & 1.25 & -0.26 & -1.00 & -5.40 & 2.64 & 0.40 & B1II & 103 & B1II \\
\hline
\end{tabular}

Notes. Columns as in Table 2.

rests entirely on the star positions on the CCD (Carraro 2011) as if they were single stars. We recall that some of them may be undetected binaries or unresolved double stars, which introduces another source of distance uncertainty due to altered colors and magnitudes. Even dealing with true single stars having MK spectral types is not enough to reduce uncertainties in distances since the MK classification and therefore the assigned $M_{V}$ may vary from author to author (see cases of different classification in Tables 2 and 3). Finally, it is evident that even among the stars located along the reddening line for B5-type stars there is still a chance of contamination by intrinsic data scatter of some reddened A-type stars. Therefore, and in an attempt to minimize wrong star inclusions, we restrict the spectroscopic parallax method to those stars in the range from B4.5- to O-types (unless otherwise stated). We are confident that, despite the errors, our large data sample will clearly reveal the main properties of the stellar populations in the region of Trumpler 27.

\subsection{Discussion on spectral types and distances of some stars}

Here we briefly discuss the nature and parameters of a few particular stars listed in Tables 2 and 3 for which distances have been derived using the spectroscopic parallax method. We focus mainly on some stars for which MK spectral types and thinprism classification comes from the literature. We also discuss briefly some potential variable stars. We refer to all these stars using the identification given in MFJ77.

Star 1: MDEW01 confirm the oldest spectral type, M0Ia, given by MFJ77. It is also a suspected variable (NSV 22849) as indicated by Samus et al. (2010). The distance of $1.8 \mathrm{kpc}$ has been derived under the assumption that it is not a variable. Additional information on this object is given later when infrared data are used.

Star 2 (LSS 4253): the old spectral type from MFJ77, O9Ia, turns out to be B0Ia according to MDEW01 with no indication about membership. This last spectral type and our new photometry locate it at almost $7 \mathrm{kpc}$ from the Sun, outside any reasonable distance limit given for Trumpler 27 in the literature.

Stars 8,10,12,19,20,21,25, and 30: they all have thinprism plates classification in MFJ77 (a classification technique that indicates whether a star is a potential OB star), and none of them were studied spectroscopically by MDEW01. In most of these cases, they were classified as potential OB stars with different degrees of accuracy as seen in Tables 2 and 3. Our procedure confirms the MFJ77 thin-prism plate classification approximation as these stars resulted from photometric spectral types $\mathrm{O} 4$, O7, O8.5, B0.5, B1.5, B1, O4, and O6, respectively.

Star 16 (LSS 4263): early spectroscopy gave O9.5 II, but changed to B0.5 Ia after MDEW01. The new spectral type and our photometry locate it at a distance of about $7 \mathrm{kpc}$.

Star 23: the spectral type determined by MFJ77, B0.5Ia, was changed to B0.7Ia after MDEW01. This star is also known as V1082 (Samus et al. 2010), a probable eclipsing variable. We derived a distance of $3.5 \mathrm{kpc}$, but if it becomes a true binary star, the distance could be reduced to $2.6 \mathrm{kpc}$ in the most favorable case of similar masses.

Star 44: this star is a B1.5Ia according to MDEW01, who have given concluding spectroscopic arguments favoring it is a supergiant star and not a giant one, as stated in MFJ77. In view of the distance of $2.5 \mathrm{kpc}$, they computed for Trumpler 27 , they assumed this star is not a cluster member, an assertion we agree with because the distance we obtain with our photometry is about $16 \mathrm{kpc}$ from the Sun. Even if we assume it is a binary star, its distance would still be more than $10 \mathrm{kpc}$ away from the Sun.

The WR stars: on one side, star 28 (WR 95) was classified WN5 by MFJ77 and WC9 by MDEW01; we adopted here this latest and modern classification. On the other side, star 105 (HDE 318016) is a WC7/WN6 following MDEW01. Conti \& Vacca (1990) reported distances of $2.8 \mathrm{kpc}$ for the WC9 and $2.4 \mathrm{kpc}$ for the WC7/WN6 that were adopted by MDEW01 in their analysis. To deal with these two complex stars, we proceeded to derive their intrinsic $(B-V)$ colors by means of the relationship between the Smith (1968) $u b v$ and $U B V$ systems as given in Lundström \& Stenholm (1984). According to the relationship given in this last paper, it was assumed $(b-v)=-0.4$ for the WC9 (WR 95) and $(b-v)=-0.3$ for the WC7/WN6 (105), while the absolute magnitudes were taken from the newest values given by van der Hucht (2001). Excesses of color obtained using the relationships of Smith (1968) were $E_{(B-V)}=1.95$ for WR 95 and 1.4 for star 105. Finally, we derived distances of 2.2 


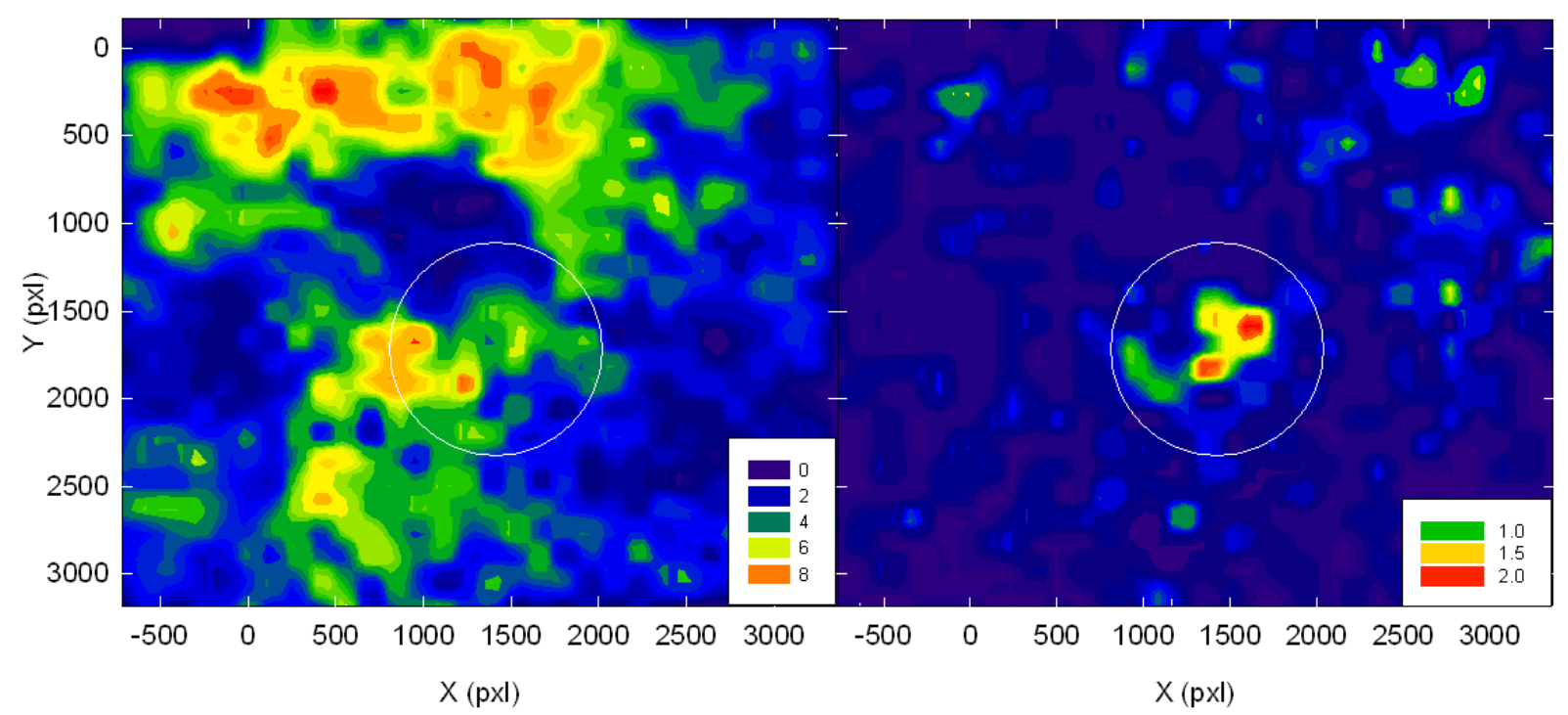

Fig. 6. The left panel is the contour plot showing the star density in the surveyed area generated approximately with 9000 stars. The insert gives the number of stars per half-minute squared box. The right panel shows the contour plot of those stars earlier than B5-type (see text). The insert as in the left panel. The $600 \mathrm{pxl}$ circle indicates the region assumed to be the core of the cluster.

for WR95 and $3.5 \mathrm{kpc}$ for star 105 that we will use in the analysis next section.

Star 107 (LSS 4257): it was classified B0V by MFJ77, but the new spectral type from MDEW01 drastically changes the point by setting this star as a supergiant of a later type, B0.5Ia. As in the previous case of star 44, they concluded this is another nonmember star. We agree since our distance sets this star at more than $10 \mathrm{kpc}$ from the Sun. Even in case of binarity, its distance would be reduced to $7.6 \mathrm{kpc}$, too far from any previous estimate of the cluster distance.

Star 102 (V925 Sco, HD159378): this is a variable star with no well-established period from $70^{\mathrm{d}}$ to more than $300^{\mathrm{d}}$ and light variation of a few tenths of a magnitude (Samus et al. 2010). Since, according to van Genderen (1980), it may not be a Cepheid variable of Pop I, we treated it as a G0 supergiant star and derived a distance of $2.4 \mathrm{kpc}$ not far from the $2.1 \mathrm{kpc}$ given by MFJ77. Parthasarathy \& Reddy (1993) placed this object at $3 \mathrm{kpc}$ from the Sun, but they gave no precise indication on the way they got their reported distance.

\section{Hunting for Trumpler 27}

We made an attempt to use proper motions in the area of Trumpler 27 from the UCAC3 data-base (Zacharias et al. 2010). The putative distance (at $2-3 \mathrm{kpc}$ from the Sun) and the crowding of the region towards the Galactic center made the exercise useless. Errors in both proper motion components are large, and no feature can be detected. This is exactly as in Moni Bidin et al. (2011), where three possibly new globular clusters are discussed toward the Galactic bulge using data from the VISTA VVV survey (Minniti et al. 2010). As in that case, proper motions are so scattered that we even restrain from showing the results.

In previous papers, we have explained (see, e.g., Vázquez et al. 2010) that instead of using star density profiles to set the cluster boundaries we prefer to look for the zone of higher star density in a contour plot. Indeed, density profiles reduce an open cluster, irregular in shape by definition, to a centrally peaked spherical stellar density distribution. On the contrary contour plots reveal details of the impact of absorption on the cluster shape and allow us to define the boundaries in a more realistic approach. The procedure works well in areas where the absorption is high, like the one in this paper. As in the case of a radial density profile, we set the size of a cluster to the area enclosed when the contour plot reaches a flat value. Notwithstanding and for the sake of completeness of the present analysis, in the next section we shall examine the infrared density profile. Meanwhile, Fig. 6 (left-hand panel) shows the contour plot $(0.5 \times 0.5$ arcmin box using the Kriging gridding method) generated with more than 9000 stars detected in the region of Trumpler 27. The full contour plot shows no star overdensity at all in the place where the cluster is assumed to be (the bright stars near the center of the mosaic in Fig. 1). Overdensities are detected north and south of the location of the cluster center, confirming what a simple eye-inspection shows in Fig. 1. Actually, the lack of a notorious overdensity right there suggests that Trumpler 27 has been detected for being a handful of bright stars well detached from the rest by dust clouds. This is better shown in the right-hand panel of Fig. 6, which displays the contour map generated only with all early-type stars of Trumpler 27 included in Tables 2 and 3 (spectral types down to B4.5). The circle in this panel has 600 pxl radio, just over 4 arcmins, and surrounds entirely the weak overdensity emerging at the position of the cluster. That is, the contour map in the right-hand panel shows that the cluster is just a handful of early stars separated by a thick dust ring from other similar groups in the region. Figure 7, left-hand panel, which displays stars in Tables 2 and 3 according to their spectral types, describes clearly the above assertion. We note that the stellar density in this region cannot be assumed to be different from that of north-west and west. The role played by the absorption is also well depicted by the contour map of $E_{(B-V)}$ excesses built in the right-hand panel of Fig. 7 with the values listed in the tables. Here we see that reddening varies in the range from 1 to more than 2 mag across the surface with higher values in a wide band from the south south-west to the north-east that separates the diverse groups seen in the left-hand panel. A comparison of the right-hand panel in Fig. 7 and the left-hand panel (all stars) in Fig. 6 suggests that a vast fraction of the A-type stars surveyed in the area may be immersed in the dust and just a few of them, together with others of later types, are likely to be located in front of the dust 


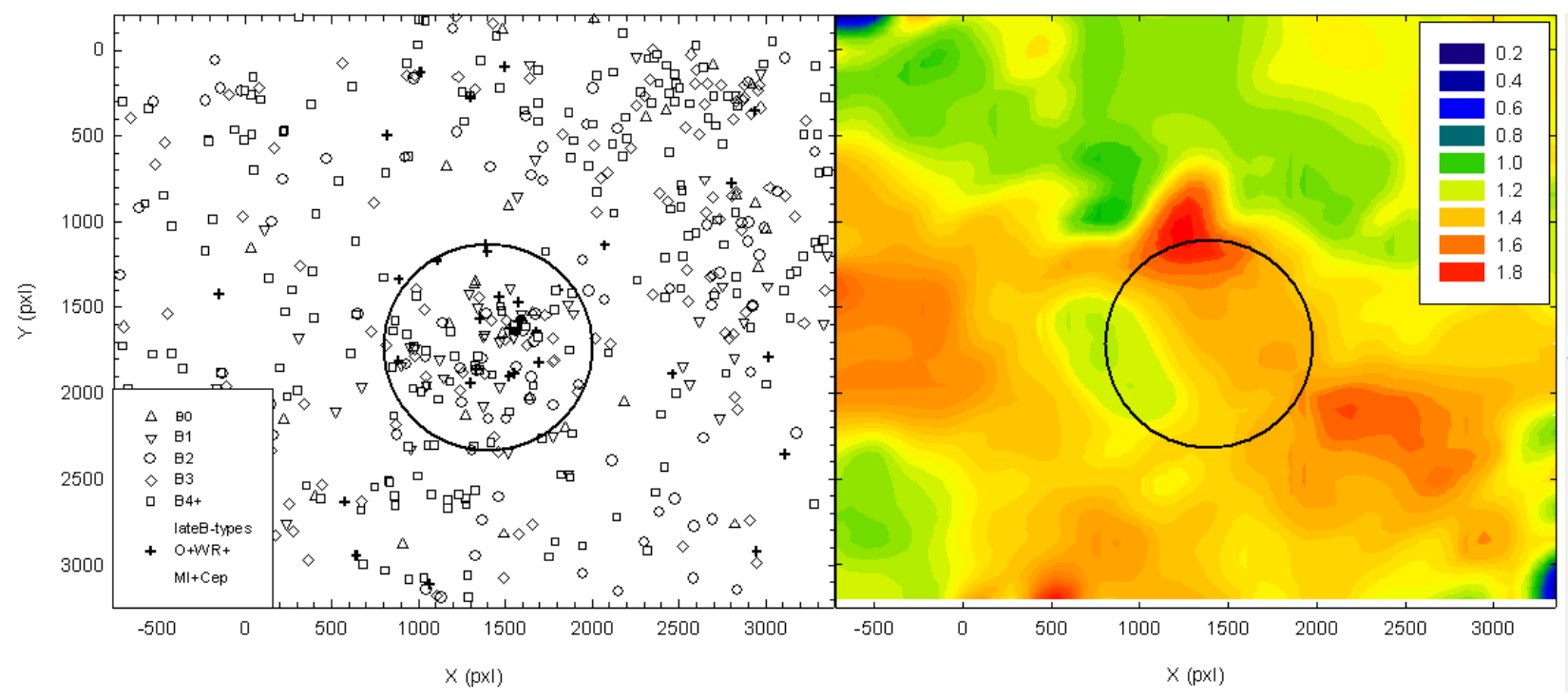

Fig. 7. The left panel stands for the spatial distribution of early-type stars according to their spectral types, shown in the insert, as explained in the text. The right panel is the contour plot of $E_{(B-V)}$ across the cluster surface. The insert gives the amount of $E_{(B-V)}$. The circle has the same meaning as in Fig. 6.

clouds. Otherwise, the overdensities seen north and south-east in the left-hand upper panel should disappear in this plot.

The zone of Trumpler 27 was included by Bitran et al. (1997) in a $\mathrm{CO}$ survey focused in a dozen degrees around the Galaxy center. The cluster is not only immersed in a region of large $\mathrm{CO}$ emission but, most interesting, it is projected against a zone of extremely high absorption as shown in map 222 made by Neckel \& Klare (1980). This alone justifies the appearance of our CCDs in Fig. 5, where potential A-F-type stars start with little absorption to end up, at $V=16$, completely immersed in the dust clouds. Likewise, the latest spectral-type stars in the field have to be necessarily nearby stars since they are intrinsically too faint and do not appear much affected by reddening.

Figure 8 traces the path of reddening with distance inside and outside the cluster boundaries. Error bars coming from Eq. (2) are also shown. It is worth briefly mentioning the impact of distance errors on our analysis. We discussed in a previous section the meaning of distance errors that are mainly based on the propagation of photometric errors, which are random in nature. Figure 8 shows the trend of our sample of stars toward the Galaxy center, and we do not see that errors can modify any of our conclusions. Looking through the core of the Trumpler 27 region, reveals that the bulk of early-type stars extends for more than $4 \mathrm{kpc}$, while others may reach $9 \mathrm{kpc}$ and even more than $15 \mathrm{kpc}$ as indicated. Something similar happens outside the 600-pixel limit, with some stars located at large distances, too. The reddening behavior shown in this figure is well in line with the above-mentioned absorption map 222 from Neckel \& Klare (1980). Map 222 and nearby ones show that absorption increases after $0.5 \mathrm{kpc}$ from the Sun, rises up to 5-6 mag in the next kiloparsec, and keeps high up to 4-5 kpc towards the Galaxy center. Our deeper and extended sample of young stars not only confirms findings of Neckel \& Klare (1980) but also states strong absorption variability all across the region, with $0.65<E_{(B-V)}<2.2$ mag meaning more than 6 mag of visual absorption. Figure 8 also shows that dust clouds are not a local phenomenon but are present for several kiloparsecs, starting near the Sun and spanning over $4 \mathrm{kpc}$ (at least) towards the center of the Galaxy.

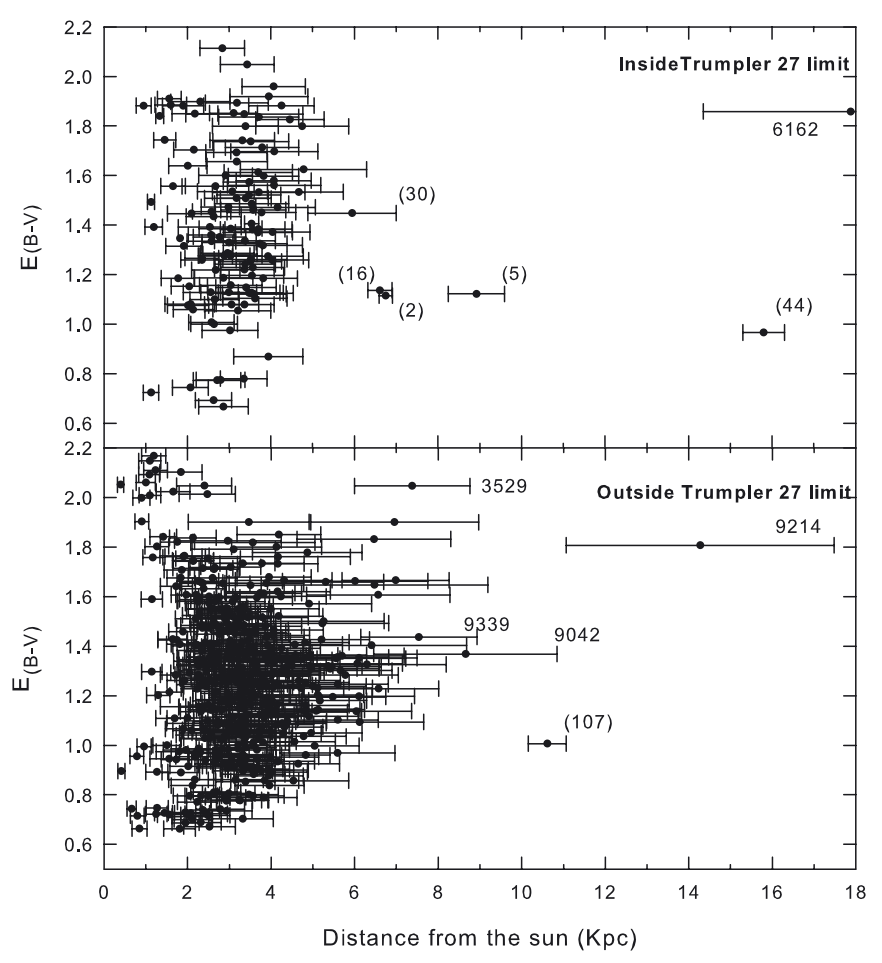

Fig. 8. Path of reddening with distance for stars inside (upper panel) and outside (lower panel) 600 pxl radius. Horizontal bars are distance errors computed from (2). Some of the distant stars are indicated. Numbers in parentheses mean the MFJ77 notation.

Histograms for different spectral types as a function of distance for both regions are shown in Fig. 8. Left-hand and righthand panels are for stars inside and outside Trumpler 27, respectively. The upper panels include O-, WR-, B0-, and late-type supergiants. Mid panels are for B1- and B2-type stars and lower panels for B3 and B4 types. For easy visualization, we restricted the plot to all the stars up to $7 \mathrm{kpc}$ from the Sun. 
In terms of confirmed spectral types, we found that 19 stars out of 126 inside the cluster limits have some type of spectral classification (MK or objective prism) from Massey and/or MFJ77, including O- and B-type stars and one WC9 star; the supergiant star N. 1 belongs to this zone. Outside the cluster radius, there are 501 early-type stars; nine of them, including the G0 Cepheid variable, have spectral analysis: one WC7/WN6, one O8.5III and one early B-type supergiant.

Inside the cluster limits, O-type stars are seen at nearly all distances. The rest of stars show a narrower distribution beyond $1.5 \mathrm{kpc}$ from the Sun. Late dwarf B-types are lacking in the first $1-1.5 \mathrm{kpc}$, probably because of dust but also because of a statistical artifact. The lack of them at larger than $4-5 \mathrm{kpc}$ is mainly due to a combination of extinction and intrinsic faintness of these spectral types. In the left-hand panels, there seems to be two peaks, one at $\sim 1-1.5 \mathrm{kpc}$ and the other higher at $\sim 3.5 \mathrm{kpc}$. The $1-1.5 \mathrm{kpc}$ peak is not far from the estimated distances by The \& Stokes (1970) and Bakker \& The (1983) for Trumpler 27, although it is a bit far, however, from the 2.1 and $2.5 \mathrm{kpc}$ given by MFJ77 and MDEW01, respectively. A more refined analysis indicates this peak contains about 20 stars, including three O-type stars at $\sim 1.5 \mathrm{kpc}$. and six other hot stars with distances between 0.9 and $1.6 \mathrm{kpc}$. The rest of the stars of photometric B-types (eight of them have evolved MK types) spread over distances from 1 to $2 \mathrm{kpc}$. Three of them with confirmed evolved MK types are in a distance range between 1.1 and $1.7 \mathrm{kpc}$. The MOIa supergiant star is placed at $1.8 \mathrm{kpc}$ in our distance estimation. However since colors of extremely red stars may be quite uncertain, it is likely located at the same distance as the others. The WC9 star, placed at $2.2 \mathrm{kpc}$, is unlikely to be related to this star group. The mean distance of all the stars in this stellar grouping (allowing for distance errors) is $1.7 \pm 0.4 \mathrm{kpc}$. This is almost $1 \mathrm{kpc}$ below the value derived by MDEW01. Compared to other distances computed for Trumpler 27, it is $0.5 \mathrm{kpc}$ less than the one estimated by MFJ77, $2.1 \mathrm{kpc}$, and about $0.5 \mathrm{kpc}$ larger than another value given by The \& Stokes (1970), $1.1 \mathrm{kpc}$, but is in good agreement with the distance published by Bakker \& The (1983), $1.6 \mathrm{kpc}$ from the Sun. However, the simple grouping by itself, with a huge distance spread, does not indicate the presence of a true open cluster, as we shall see later on. If we look outside the region of the location of Trumpler 27 in Table 3, we find only 35 stars sharing a similar distance range.

Regarding the second star peak at $\sim 3.5 \mathrm{kpc}$ seen in the direction to Trumpler 27 core, it is quite far from the distance 2.1 and $2.5 \mathrm{kpc}$ given for Trumpler 27 by MFJ77 and MDEW01. Moreover, it is wider than the first one and tends to show some kind of coincidence with the evident peak of stars outside the cluster region approximately at the same distance (see righthand panels in Fig. 9). In this distance range from 3 to $3.5 \mathrm{kpc}$, there are more than 110 stars outside the potential cluster core, including three stars of $\mathrm{O}$ types and one B-type supergiant out of 27 stars with MK types. The WC7/N6 and the G0I stars, both at $2.4 \mathrm{kpc}$ approximately, are probably not related to this second peak of stars, either.

The histograms of Fig. 9 are conclusive in the sense that early-type stars are found at any distance inside and outside the cluster region. That is why, in our opinion, different authors arrive at different conclusions about members and distances of this putative open cluster. Additional proof is provided by the observed CMDs of early-type stars found inside and outside the cluster boundaries shown in Fig. 10. Here, stars have been identified with different symbols according to their distances, leading to considerations that any combination of them may produce a believable CMD. In particular, the stars in the 3-4 kpc range are
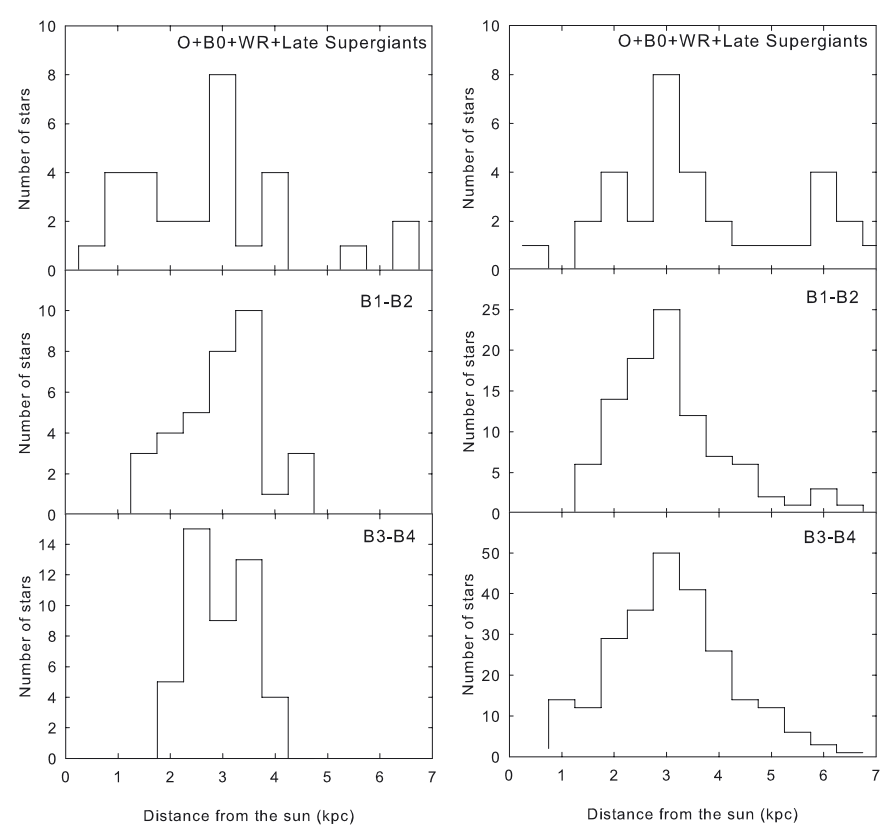

Fig. 9. Histograms of stars for spectral types. Left panels for those inside the cluster limits and right panels for those stars outside the cluster (see text for explanation).

responsible for the left-hand envelope and high density of both CMDs, while the most dispersed stars are those belonging to the $2-3 \mathrm{kpc}$ range. Inside the limits, where Trumpler 27 is assumed to exist, the upper part of the CMD is composed by stars as near as $2 \mathrm{kpc}$ and as far as more than $5 \mathrm{kpc}$ from the Sun.

From the above analysis, we conclude that the evidence is not conclusive enough to clearly establish the true nature and location of Trumpler 27. There is no doubt here that we are dealing with a diffuse young population of stars distributed all along the line of sight with some stellar clumps, including all types of bright stars.

\section{Infrared information}

Infrared data provided by the GLIMPSEII spring'08 (highly reliable) catalog was cross-correlated with our optical catalog to get 2MASS JHKs and IRAC 3.6, 4.5 and $8 \mu \mathrm{m}$ data. In particular, $J H K$ s data was used to perform an additional search for Trumpler 27, as can be seen in Fig. 11 (down to $J=16 \mathrm{mag}$ ). Using all stars in the GLIMPSEII catalog that lie inside the boundaries of our observed frames, a radial density profile was constructed as shown in the upper left-hand panel of Fig. 11. The profile gives the number of stars per square arcmin computed as the number of stars found in concentric annuli 1 arcmin wide, starting at the current adopted cluster coordinates given in Sect. 1. Error bars are Poisson errors (computed as $\sqrt{N}$ with $N$ the number of stars per square arcmin). The panel confirms that the cluster is only an artifact produced by the evident dust ring around the center, as shown by the pronounced dip at 3 arcmin from the center. This is the same shown in Fig. 6, left-hand panel. Apart from the dip, the star density profile is essentially plain, within the Poisson errors, showing no evidence of a star clustering.

Close to star N. 1 and 1b (MDEW01 numbering), there exists the maser source OH355.1-0.76 measured by Knapp et al. (1989) in their circumstellar CO emission survey. Unfortunately, they give no further information on this object, and it was discarded 


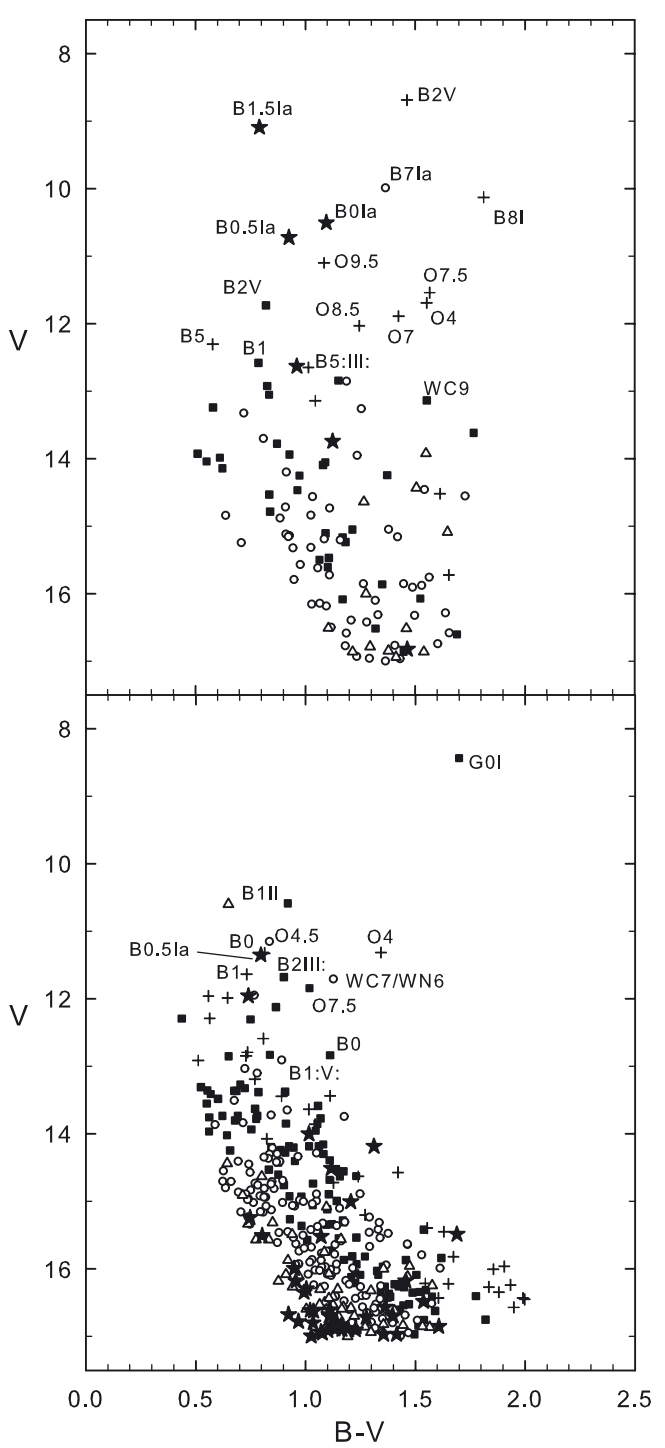

Fig. 10. CMDs of stars ordered by distance. Symbols are: (+) stars from 0 to $2 \mathrm{kpc},(\square)$ for $2-3 \mathrm{kpc},(\circ)$ for $3-4 \mathrm{kpc},(\Delta)$ for $4-5 \mathrm{kpc}$ and $(\star)$ stars beyond $5 \mathrm{kpc}$. Upper panel, all stars above B5- spectral types in Trumpler 27 region. Lower panel, idem above for stars outside.

due to the strong contamination caused by Galactic CO emission over a wide range of velocity. We assume that the supergiant star N. 1 is probably the optical counterpart of this object because it, in turn, is also a suspected variable (Samus et al. 2010). Across the area surveyed in this article, we found optical counterparts of two YSO candidates (Nos. 5338 and 5269 in our numbering, corresponding to 2MASSJ17362441-3331289 and 2MASSJ17362515-3325521 sources, respectively) and another star (N. 1796, our numbering) with huge infrared excess.

The upper right-hand panel in Fig. 11 is the overall $(J-H)$, $(H-K)$ two-color diagram. The solid black line indicates the intrinsic relation from Koornneef (1983), and the two gray dashed lines indicate the 6 mag reddening vectors $E_{(J-H)} / E_{(H-K)}=1.7$ for early- and late-type stars. We have superimposed stars earlier than B5 inside 600 pxls radius (white squares) and outside these limits (red squares). Despite the fact that data scatter is strong and typical infrared errors are large, this diagram confirms that stars in the region of Trumpler 27 are affected by strong variable reddening and shows no indication of a clear cluster sequence. Indeed, the spread of the data inside $600 \mathrm{pxl}$ is similar to the
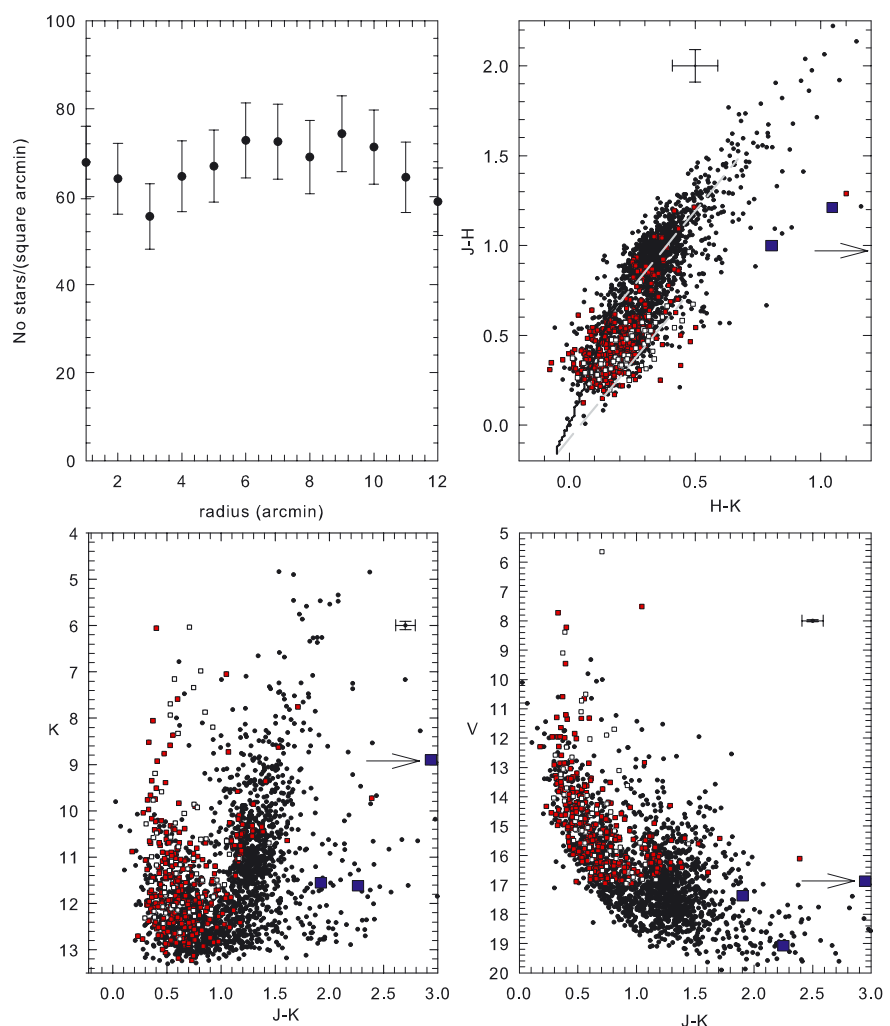

Fig. 11. Radial density profile in the region of Trumpler 27, upper left panel. Bars are Poisson errors. The $(J-H),(H-K)$ color-color diagram of all stars in the region is shown in the upper right panel. White and red squares are stars in Tables 2 and 3, respectively. The cross indicates the average color errors. Large blue squares give the position of the YSO candidates, and the arrow points out the position of the star N. 1796 outside the diagram limits. Solid black line is the intrinsic relation from Koornneef (1983); the two gray dashed lines are color excesses relations for $A_{V}=6 \mathrm{mag}$ for early- and late-type stars. The lower left and right panels show the $K,(J-K)$ and $V,(J-K)$ color-magnitude diagrams. Symbols as in the upper right panel.

one shown by the other early stars outside this limit. Large blue squares and the arrow show the position of the YSO candidates mentioned above and of star N. 1796. It seems that more objects in the zone exhibit large infrared excesses. The other two lower panels are the $K,(J-K)$ and $V,(J-K)$ CMDs. These two diagrams show the same pattern found in Fig. 4 in the sense that no clear cluster sequence emerges from them and no evidence of a pre-main sequence is revealed. The illusory vertical strips seen in the $K,(J-K)$ are composed by stars at very different distances inside the 600 pxl radius and also outside that limit. Actually, with no other information available to impose boundary conditions (as the one provided by the CCD in Fig. 4 and MK types), it would be possible to confuse the vertical strip with the upper sequence of an unreal cluster. For the sake of completeness, we have also plotted $3.6,4.5$, and $8 \mu \mathrm{m}$ data in the adequate diagrams (not shown to save space) and, again, no evidence for a cluster was found in any of them.

\section{The region toward $I=355^{\circ}$ and the inner spiral structure of the Galaxy}

The presence of over 600 stars of very early spectral types in a small sky area of a quarter of square degree located in a continuous range of distance up to at least $7 \mathrm{kpc}$ from the Sun 
G. Perren et al.: Photometric distances to young stars in the inner Galactic disk. II.
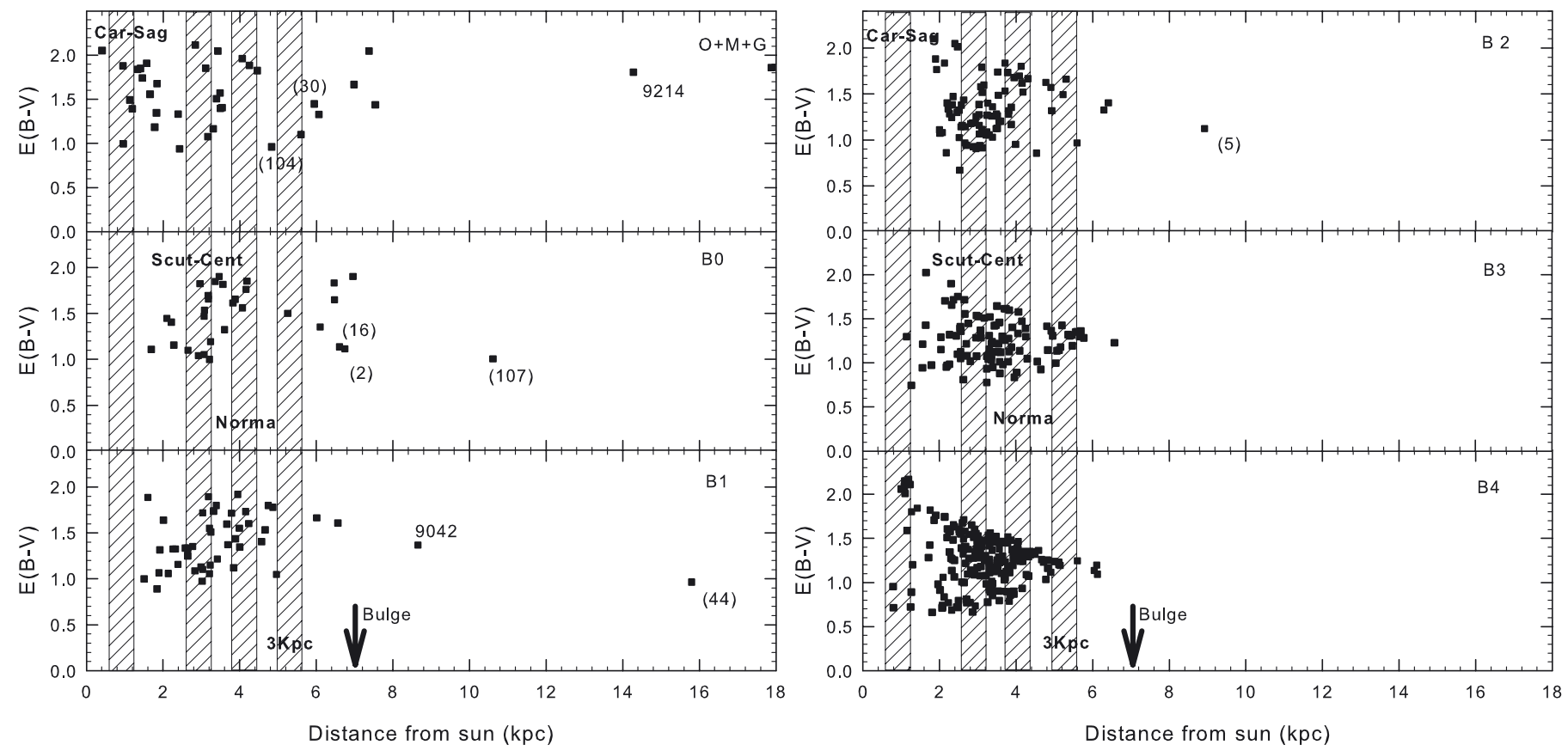

Fig. 12. Path of reddening with distance according to the star spectral type. The approximate position and width of the inner spiral arms is shown by dashed columns; the probable beginning of the bulge is indicated by the arrow. Numbers in parentheses, as in Fig. 8.

is an exciting result of our survey, which connects this young diffuse population to the inner Galactic structure. In the traditional model of four arms for the Galaxy (Russeil 2003; Vallée 2005) moving from the Sun location inwards, in the region of Trumpler 27, there should be Sagittarius-Carina arm, ScutumCrux arm, Norma, and the Near $3 \mathrm{kpc}$ arms at 0.6,3, 4, and $5 \mathrm{kpc}$ respectively. In the proposed recent face-on view of our Galaxy (Churchwell et al. 2009), the cross with Sagittarius-Carina arm should happen at $1.6 \mathrm{kpc}$. In particular, this arm looks weak and narrow, i.e., it is tiny and not very prominent. In the same sketch, the Scutum-Crux arm is located at $3.2 \mathrm{kpc}$ and becomes the most prominent feature of the inner Galactic structure. The Norma arm is another tiny structure intersected at $4.4 \mathrm{kpc}$ and, finally, the Near $3 \mathrm{kpc}$ arm at over $5 \mathrm{kpc}$ is the less pronounced of all the features. A recent map of the Milky Way has been presented by Lépine et al. (2011), where the traditional four-arm structure is abandoned and replaced by polygonal arms described by molecular CS associated with IRAS sources. Given the complex structure proposed by Lépine et al. (2011), we prefer to compare our findings with the traditional description (e.g., Russeil 2003).

In terms of stellar components, mainly O-B3-type stars and open clusters associated with the grand design spiral structure, the information is scarce as shown by Russeil (2003). The OBtype star catalog recently compiled by Reed (2003) was used by Carraro (2011) to highlight the spiral structure around the Sun. Figure 1 in Carraro (2011) demonstrates that only O-type stars show some sort of clear coincidence with spiral arms of the Galaxy while B-type stars, on the contrary, show a smooth distribution up to the reach of the survey made by Reed (2003). Carraro (2011) also emphasizes the lack of coincidence of the star sample (O-plus B-types) with the trace of Scutum-Crux. In the direction we are dealing with, the Reed (2003) catalog scarcely reaches $5 \mathrm{kpc}$ from the Sun, while the number of confirmed (spectroscopically) O-type stars by Maíz-Apellániz et al. (2004), complete only to $V=8 \mathrm{mag}$, indicates that very few confirmed stars of this type are found in the direction where Trumpler 27 is located.
Returning to our data sample, we have included stars of Tables 2 and 3 in the distance vs $E_{(B-V)}$ plot of Fig. 12 up to $18 \mathrm{kpc}$ from the Sun. In the figure, we also have marked the distances to which the spiral arms of the Galaxy should approximately be located, according to the current accepted model of four arms and their respective widths. We briefly recall that while arm separation is of the order of $3 \mathrm{kpc}$, the width of the arms is quite uncertain. We set arm widths in Fig. 12 using the value of about $1 \mathrm{kpc}$ given by Vallee (2005). Accepting that Sagittarius-Carina is at less than $1 \mathrm{kpc}$ in the $355^{\circ}$ direction, we found very few stars associated with this spiral arm, mostly $\mathrm{O}$ and B3-B4, while the bulk of young stars tend to gather at the distance where Scutum-Crux and Norma arms are set. The plots in Fig. 12 are in line with Fig. 1 shown by Carraro (2011) in the sense that very young stars do not define conclusively the trace of inner spiral arms but are distributed evenly across several kiloparsecs toward the center of the Galaxy. This is a singular difference with radio observations. In fact, maps of the HI and $\mathrm{CO}$ contributions suggest that these arms are discrete and well-outlined structures, Scutum-Crux being a major one moving toward the inner Galaxy.

The recent study by Dame \& Thaddeus (2008) collected specific and irrefutable evidence of the presence of the distant counterpart of the Near $3 \mathrm{kpc}$ arm based in $\mathrm{CO}$ and previous observations in HI. The authors estimated that distances from the Sun for both arms are 5.1 and $11.8 \mathrm{kpc}$ for the Near $3 \mathrm{kpc}$ arm and the Far $3 \mathrm{kpc}$ arm, respectively. It is worth mentioning that stars at all spectral types (also one O-type) can be found in or close to the Near $3 \mathrm{kpc}$ arm in Fig. 12. If the tendency in our sample is correct, for the first time we are looking at components of a very recent star formation process there. From the point of view of our findings, the relation between the young population and the Near $3 \mathrm{kpc}$ arm is not controversial. As Dame \& Thaddeus (2008) show, the Near $3 \mathrm{kpc}$ arm extends mostly below the Galactic plane at the location of Trumpler 27, and thus part of the young stars in our sample may be spatially connected with this arm. There are stars even farther in this region (at more 
than $8 \mathrm{kpc}$ ) that have no chance to be related to the Far $3 \mathrm{kpc}$ arm, since it extends mainly above the Galactic plane.

From our study, based on an extended sample of early-type stars detected photometrically, we find it hard to establish the relation of the inner spiral arms with our stellar population. While the gas component is expected to trace the path of every inner arm by means of discrete structures of molecular $\mathrm{CO}$ and HI, stellar distances of young stars do not. Figure 12 allows us to assure that the trend of the young diffuse population extends all along $7 \mathrm{kpc}$ towards the center of the Galaxy, but by no means can we specify the position and distance from the Sun of each arm. This difficulty arises from a combination of distance uncertainties with geometrical circumstances. In fact, despite the fact that the process of star formation is active and vigorous, it is hard to relate it to specific patterns of spiral structure because of a) the tight packing of four arms in such a short distance range, and b) our line of sight on the plane is almost at right angles with the spiral structure.

All the circumstances mentioned above are reflected in the radio observation domain. Very recently, Green et al. (2011) have been studying the distribution of methanol masers in the inner Galaxy covering the longitude range from $-28^{\circ}$ to $+28^{\circ}$. In particular, in the direction of our analysis, they found an enhancement of methanol sources that are comprehensible only as a superposition of contributions from large Galactic structures confused by intervening spiral arms along the line of sight. This difficulty is exactly the same that we found in this paper.

\section{Conclusions}

This is the first time that an $U B V I$ CCD photometric study is carried out in the region of the open cluster Trumpler 27 towards $l=355^{\circ}$. In order to determine the properties of the stellar population, we have applied well-known broad-band photometric techniques. Our goal has been twofold: on one side, to recognize the main parameters of the cluster, if it exists, since several observers in the past arrived at different conclusions, and, on the other side, to analyze the field star population present in the region, since the same studies underlined the presence of a large number of bright stars that have no relation with the cluster itself.

An open cluster is, in essence, a decreasing sequence of gravitationally bound stellar masses well confined in a volume of space. In the case of nearby open clusters, the analysis of proper motions and radial velocities becomes a powerful tool to assess the cluster membership on any star found in the field. The way to assess if a star belongs to distant clusters is by relying on a spectrophotometric study focused on some sort of stellar overdensity detected against the sky background. In the zone of Trumpler 27 , there is neither reliable proper motions, given the distances of the stars in the region, nor appreciable star overdensity, even when using infrared data, as shown in Fig. 11, upper left-hand panel.

From a spectrophotometric point of view, we cannot confirm or rule out definitely the existence of the cluster since results of our analysis based on separating early-type stars from field stars are not conclusive at all. That is, CMDs showing the same pattern can be constructed with stars at different distances from the Sun neighborhood all the way to the Galactic center. Massive stars of O-type, evolved B-types, M- and G- supergiants plus two WR stars are found at all distances and widely separated angularly from each other, making it unlikely to connect them from an evolutionary point of view. The different CMDs in optical and infrared are typical of very young star groups superposed along the line of sight resembling, e.g., OB associations. This could be the reason for the age spread detected by MDEW01, since the entire star group associated historically with Trumpler 27 is composed of stars that did not form in a same event and do not share the same spatial location.

Based on our data, it is difficult to assess the existence of Trumpler 27 as a star cluster using the parameters of age, reddening, and distance, suggested by previous investigations. In addition, we were unable to find in our diagrams a star sequence composed by faint stars that could be related beyond doubt with potential massive members of Trumpler 27. This is a serious obstacle, and we find it unlikely that photometry alone could solve the question in the future. Certainly, the controversy may be settled by means of an exhaustive spectroscopic study able to reach also faint stars. An interesting point to interpret, in terms of star formation, is the lack of any evident HII region in the zone, as expected in a region of massive star formation. WR- and O-Type stars are usually injecting a tremendous amount of ultraviolet photons in the surrounding gas that should be seen in emission, but there are no clear traces of emitting gas.

As far as the diffuse young star population is concerned, our star sample shows an even distribution in the direction $l=355^{\circ}$. This suggests that early-type stars define a continuous superposition of stars with no density peaks from one arm to the next, except for the prominent clump at the location of the Scutum-Crux arm, as seen in Fig. 9.

The findings of this paper can be connected with the ones from Carraro (2011) at $l=314^{\circ}$. Firm evidence was found there of a young stellar population associated with Carina-Sagittarius and Scutum-Crux arms. Moreover, the existence of an even star distribution in the direction $l=314^{\circ}$, where arms are more open than in the Galactic center direction, was found. This confirmed the outcome of old (e.g., Muzzio \& Levato 1980, and references therein) and more recent (Carraro \& Costa 2009; Baume et al. 2009) studies that provided robust optical evidences of their existence and their potential as spiral arm indicators.

However, in the present study, which looks towards the center of the Galaxy, four spiral arms are closely packed in only $5 \mathrm{kpc}$ from the Sun. Therefore, the task of disentangling one structure from the next is more cumbersome and would require the support of an improved radio trigonometric parallax method to measure accurate distances and proper motions of obscured star-forming regions in the Milky Way (Brunthaler et al. 2011).

Meanwhile, we believe it is of extreme value to continue our program, which aims at studying the inner disk stellar populations using $U B V$ and infrared photometry by extending it to other absorption windows, as in the case of Trumpler 27. Young stellar populations can be very well tracked using $U B V$ photometry, and when combined with findings from radio observations, a better picture of large structures can certainly be achieved.

Acknowledgements. G. Carraro expresses his gratitude to the Las Campanas Observatory staff, and in particular to Patricio Pinto for the excellent support during various observing runs. We also thank Sandy Strunk for reading carefully the manuscript. G. Perren and R. A. Vázquez acknowledge the financial support from the CONICET PIP1359. This research made use of the NASA/IPAC Infrared Science Archive, which is operated by the Jet Propulsion Laboratory, California Institute of Technology, under contract with the National Aeronautics and Space Administration. We acknowledge useful and constructive comments from an anonymous referee that greatly allowed us to improve the manuscript.

\section{References}

Ahumada, J. A., Giorgi, E. E., Solivella, G.,\& Vázquez, R. A. 2011, MNRAS, 415,1355

Baume, G., Carraro, G., \& Momany, Y. 2009, MNRAS, 398, 221

Bakker, R., \& The, P. S. 1983, A\&AS, 52, 27

Bitran, M., Alvarez, H., Bronfman, L., May, J., \& Thaddeus, P. 1997, A\&AS, 125,99 
G. Perren et al.: Photometric distances to young stars in the inner Galactic disk. II.

Brunthaler, A., Reid, M. J., Menten, K. M., et al. 2011, Astron. Nachr., 332, 461 Carraro, G. 2011, A\&A, 536, A101

Carraro, G., \& Costa, E. 2009, A\&A, 493, 71

Carraro, G., Vázquez, R. A., Moitinho, A., \& Baume, G. 2005, ApJ, 630, L153

Carraro, G., Moitinho, A., Zoccali, M., Vázquez, R. A., \& Baume, G. 2007, AJ, 133,1058

Carraro, G., Vázquez, R. A., Costa, E., Perren, G., \& Moitinho, A., 2010, ApJ, 718,683

Churchwell, E., Babler, B. L., Meade, M. R., et al. 2009, PASP, 121, 230

Conti, P. S., \& Vacca, W. D. 1990, AJ, 100, 431

Dame, T. M., \& Thaddeus, P. 2008, ApJ, 683, L143

Hamuy, M., Folatelli, G., Morrel, N. I., et al. 2006, PASP, 118, 2

Hiltner, W. A., \& Johnson, H. L. 1956, ApJ, 124, 367

Green, J. A., Caswell, J. L., McClure-Griffiths, N. M., et al. 2011, ApJ, 733, 27

Knapp, G. R., Sutin, B. M., Ellison, B. N., et al. 1989, ApJ, 336, 822

Koornneef, J. 1983, A\&A, 128, 84

Landolt, A. U. 1992, AJ, 104, 340

Lépine, J. R. D., Roman-Lopes, A., Abraham, Z., Junqueira, T. C., \& Mishurov, Yu. N. 2011, MNRAS, 414, 1607

Lundström, I., \& Stenholm, B. 1984, A\&AS, 58, 163

Maíz-Apellaíniz, J., Walborn, N. R., Galué, H., \& Wei, L. H. 2004, ApJS, 151, 148

Marraco, H. G., \& Rydgren, A. E. 1981, AJ, 86, 62

Massey, P., DeGioia-Eastwood, K., \& Waterhouse, E. 2001, AJ, 121, 1050
McSwain, M. V., \& Gies, D. R. 2005, ApJS, 161, 118

Minniti, D., Lucas, P. W., Emerson, J. P., et al. 2010, New Astron., 15, 433

Moffat, A. F. J., FitzGerald, M. P., \& Jackson, P. D. 1977, ApJ, 215, 106

Moitinho, A., Vázquez, R. A., Carraro, G., et al. 2006, MNRAS, 368, L77

Moni Bidin, C., Mauro, F., Geisler, D., et al. 2011, A\&A, 535, A33

Muzzio, J. C., \& Levato H. O. 1980, PASP, 92, 36

Neckel, Th., \& Klare, G. 1980, A\&AS, 42, 281

Parthasarathy, M., \& Reddy, B. E. 1993, ASIB, 21, 619

Patat, F., \& Carraro, G. 2001, MNRAS, 325, 1591

Reed, C. B. 2003, AJ, 125, 2531

Russeil, D. 2003, A\&A, 397, 133

Samus, N. N., Kazarovets, E. V., Kireeva, N. N., Pastukhova, E. N., \& Durlevich, O. V., 2010, Odessa Astron. Publ., 23, 102

Schmidt-Kaler, T. 1982, Landolt-Börnstein, Group VI, Vol. 2b, Stars and Star Clusters (Berlin: Springer), 15

Skrutskie, M. F., Cutri, R. M., Stiening, R., et al. 2006, AJ, 131, 1163

Smith, L.F. 1968, MNRAS, 140, 409

Stetson, P. B. 1987, PASP, 99, 191

The, P. S., \& Stokes, N. 1970, A\&A, 5, 298

Vallée, J. 2005, AJ, 130, 569

van der Hucht, K. A. 2001, New Astron. Rev., 45, 135

van Genderen, A. M. 1980, A\&A, 88, 77

Vázquez, R. A., May, J., Carraro, G., et al. 2008, ApJ, 672, 930

Zacharias, N., Finch, C., Girard, T., et al. 2010, AJ, 139, 2184

Page 14 is available in the electronic edition of the journal at http://www . aanda.org 
Table 1. UBVI photometric observations of Trumpler 27 and standard stars.

\begin{tabular}{|c|c|c|c|c|c|c|}
\hline Target & RA & Dec & Date & Filter & Exposure (s) & Airmass $(X)$ \\
\hline Field-1 & $17: 36: 20.79$ & $-33: 28: 02.8$ & 2006 May 31 & $\begin{array}{l}U \\
B \\
V \\
I\end{array}$ & $\begin{array}{c}20,200,2 \times 1500 \\
10,100,2 \times 1200 \\
5,10,100,900 \\
5,10,2 \times 100\end{array}$ & $\begin{array}{l}1.02-1.05 \\
1.00-1.00 \\
1.14-1.16 \\
1.08-1.10 \\
\end{array}$ \\
\hline Field-1 & $17: 36: 20.79$ & $\begin{array}{l}-33: 28: 02.8 \\
-33: 28: 02.8\end{array}$ & 2006 Jun. 31 & $\begin{array}{l}B \\
V \\
I \\
U \\
B \\
V \\
I\end{array}$ & $\begin{array}{l}40 \\
20 \\
20 \\
60 \\
30 \\
30 \\
30\end{array}$ & $\begin{array}{l}1.00-1.01 \\
1.00-1.01 \\
1.00-1.01 \\
1.00-1.01 \\
1.00-1.01 \\
1.00-1.01 \\
1.00-1.01 \\
\end{array}$ \\
\hline Field-1 & $17: 36: 20.79$ & $-33: 28: 02.8$ & 2010 May 08 & $\begin{array}{l}U \\
B \\
V \\
I\end{array}$ & $\begin{array}{c}15,90,300,1500 \\
5,45,300,1200 \\
2 \times 5,120,900 \\
2 \times 5,30,600\end{array}$ & $\begin{array}{l}1.02-1.03 \\
1.06-1.10 \\
1.10-1.15 \\
1.01-1.01\end{array}$ \\
\hline Field-2 & 17:36:54.79 & $-33: 28: 02.8$ & 2010 May 08 & $\begin{array}{l}U \\
B \\
V \\
I\end{array}$ & $\begin{array}{c}15,90,300,1500 \\
5,45,300,1200 \\
2 \times 5,120,900 \\
2 \times 5,30,600\end{array}$ & $\begin{array}{l}1.01-1.01 \\
1.03-1.04 \\
1.12-1.14 \\
1.05-1.08\end{array}$ \\
\hline Field-3 & $17: 35: 47.79$ & $-33: 28: 02.8$ & 2010 May 08 & $\begin{array}{l}U \\
B \\
V \\
I\end{array}$ & $\begin{array}{c}15,90,300,1500 \\
5,45,300,1200 \\
2 \times 5,120,900 \\
2 \times 5,30,600\end{array}$ & $\begin{array}{l}1.05-1.11 \\
1.11-1.18 \\
1.36-1.42 \\
1.20-1.28 \\
\end{array}$ \\
\hline Field-1 & 17:36:20.79 & $-33: 28: 02.8$ & 2011 Jun. 03 & $\begin{array}{l}U \\
B \\
V \\
I\end{array}$ & $\begin{array}{c}60,180 \\
30,120 \\
10,60 \\
10,60\end{array}$ & $\begin{array}{l}1.00-1.01 \\
1.00-1.01 \\
1.00-1.01 \\
1.00-1.01\end{array}$ \\
\hline Mark A & 20:43:55.09 & $-10: 45: 38.0$ & 2011 Jun. 03 & $\begin{array}{l}U \\
B \\
V \\
I\end{array}$ & $\begin{array}{c}180,2 \times 240 \\
120,2 \times 180 \\
60,2 \times 90 \\
60,2 \times 90\end{array}$ & $\begin{array}{l}1.07-1.60 \\
1.07-1.56 \\
1.07-1.53 \\
1.06-1.51\end{array}$ \\
\hline PG 1323 & $13: 25: 38.91$ & $-08: 50: 05.6$ & 2011 Jun. 03 & $\begin{array}{l}U \\
B \\
V \\
I\end{array}$ & $\begin{array}{c}3 \times 180 \\
3 \times 120 \\
3 \times 60 \\
3 \times 60\end{array}$ & $\begin{array}{l}1.08-1.73 \\
1.08-1.77 \\
1.07-1.82 \\
1.07-1.85\end{array}$ \\
\hline PG 1633 & $16: 35: 24.91$ & $09: 47: 39.8$ & 2011 Jun. 03 & $\begin{array}{l}U \\
B \\
V \\
I\end{array}$ & $\begin{array}{c}2 \times 180,240 \\
2 \times 120,180 \\
3 \times 60,90 \\
2 \times 60,90\end{array}$ & $\begin{array}{l}1.29-1.83 \\
1.29-1.88 \\
1.29-2.02 \\
1.29-1.97\end{array}$ \\
\hline
\end{tabular}

